\title{
'STISIM-Drive' Meets ‘MotorcycleSim': Using Driving Simulation Software to Develop a Unique Motorcycle Simulator for Rider Behavior Research
}

\author{
Alex W. Stedmon ${ }^{1}$, David Crundall ${ }^{2}$, Elizabeth Crundall $^{2}$, Rose Saikayasit ${ }^{1}$, \\ Editha van Loon ${ }^{2}$, Alex Irune ${ }^{3}$, Patrick Ward ${ }^{2}$, and Neil Greig ${ }^{4}$ \\ ${ }^{1}$ Centre for Motorcycle Ergonomics \& Rider Human Factors, University of Nottingham, UK \\ alex.stedmon@nottingham.ac.uk \\ ${ }^{2}$ School of Psychology, University of Nottingham, UK \\ ${ }^{3}$ Dimax Technology Ltd, Nottingham, UK \\ ${ }^{4}$ The Institute of Advanced Motorists, IAM House, London, UK
}

\begin{abstract}
In order to compare groups of road users who have fundamentally different skills, attitudes and behaviours, one of the first in-depth motorcycle simulation studies of its kind was conducted. The project was developed using the innovative 'MotorcycleSim' simulator designed and built at the University of Nottingham. The simulator is a research tool to investigate aspects of motorcycle ergonomics and rider human factors and is the first of its kind in the world to incorporate 'STI-SIM Drive' software that allows motorcyclists to ride a full size motorcycle and interact with a virtual riding environment (VRE). To build a simulator that was both fit for the purpose of research and provided the desired levels of fidelity associated with real world riding, a user-centred design process was adopted from the outset (based in principles of ISO:13407).
\end{abstract}

Keywords: transport simulation, motorcycles, rider behavior.

\section{Introduction}

Capturing road user experiences in the real world is extremely valuable and underpins the applied nature of ergonomics within human-machine interaction [1]. However, due to practical, safety and ethical issues surrounding real world research, simulators offer the potential to develop alternative research tools [2]. Simulators offer a level of abstraction from the real world by providing an artificial environment in which users can experience characteristics of a real system [2]. Whilst there are limits to the degree of realism that can be achieved in simulators, if they are designed well, they offer high levels of ecological validity [1]. Underpinning this is a key human factors question regarding the level of realism required for a simulator to serve its intended purpose (e.g. training, research, product development, etc) based on a fundamental understanding of user-centred design principles [3].

From the outset, a detailed user-centred design methodology was adopted based on principles of ISO:13407 [4]. Without such an approach there is always a risk of specifying a solution that fails to support the users' understanding of the target 
application [5]. A questionnaire survey incorporated 13 characteristics that participants rated on a 5-point scale [1]. The results indicated that the riders required the simulator to lean like a real motorcycle, have acceleration and braking effects and for the controls to work in a realistic manner. From additional comments that were made, riders highlighted the level of realism the simulation software might offer and how other traffic and road conditions could be incorporated into the virtual riding environment (VRE). Furthermore, a number of riders felt the simulator should be capable of replicating vibration and allow for riding with a passenger. The user requirements formed the basis of the development of MotorcycleSim as well as highlighting development routes for the future [1].

\section{MotorcycleSim: An Overview}

MotorcycleSim has been built using a full size and fully equipped Triumph Daytona 675 motorcycle (kindly supplied by Triumph Motorcycles). The existing motorcycle controls (e.g. throttle, brake lever, brake pedal, gear selector, clutch lever) were modified or adapted to work with the simulation software in a realistic manner. MotorcycleSim uses commercially available 'STISIM-Drive' software which is an industry leader and has a worldwide support community. Although primarily a driving simulation package, the software can be used to build interactive VREs that allow for strictly controlled experimental repeatability in a laboratory setting as riders experience identical scenarios which are not possible on the road [3].

Within the 'STISIM-Drive' software it is possible to change vehicle characteristics to alter the simulated handling and performance profile of the motorcycle. Given focus of the software on driving applications, the vehicle characteristics have been fine-tuned to mimic a motorcycle and to a large extent this has been achieved by 'fooling' the software that the vehicle is a small and lightweight car with the acceleration and braking characteristics of a motorcycle. Alongside this, bespoke software and engineering solutions were developed to integrate the motorcycle hardware with the simulation software [2].

The visual representation (projected onto a large screen in front of the rider) can be manipulated in a number of ways:

- enhanced acceleration and braking effects are presented by altering the degree of dynamic pitch in the visual scene as the motorcycle accelerates and slows down;

- the scenery tilts as the rider steers in a particular direction to enhance the perception into leaning into a corner

- the rider's field of view can be increased to take account of peripheral visual cues.

MotorcycleSim can be used in static or dynamic modes. In the dynamic mode the motorcycle tilts as the bespoke software takes data for speed and the steering angle in order to calculate an angle of lean (Figure 1). 


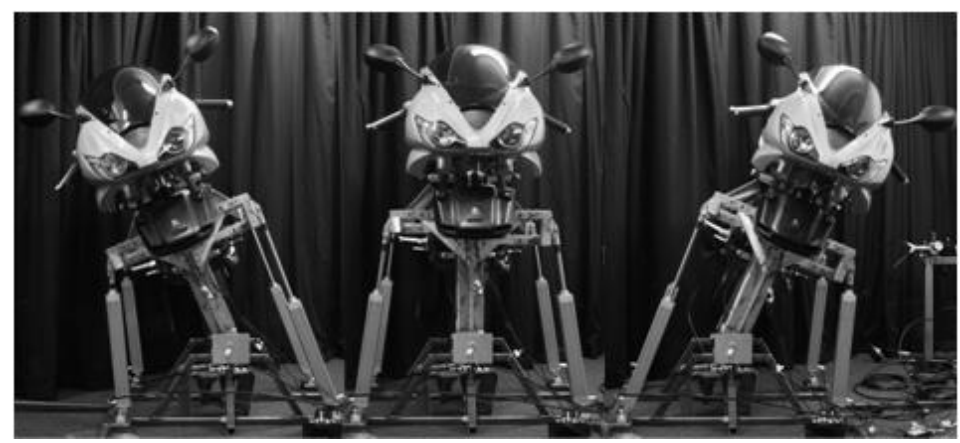

Fig. 1. The unique titling action of MotorcycleSim

MotorcycleSim, has been designed to offer four different simulation modes: static simulation with 'STISIM-Drive'; cornering/leaning training; cornering/leaning training with 'STISIM-Drive'; and an advanced dynamic model with 'STISIM-Drive' that aims to offer a realistic riding experience by utilising a comprehensive dynamics model to control the leaning of the motorcycle as the rider navigates the VRE.

\section{Integrating MotorcycleSim with 'STISIM-Drive'}

In order to interact with the 'STI-SIM Drive' software, the mechanical movement of the throttle, braking, gear selection and steering angle of the motorcycle was interfaced with a standard automotive games controller unit (Figure 2). This solution was used as no precedent for a motorcycle controller exists with 'STISIM-Drive' and as such, a novel solution had to be explored:

- throttle - the throttle on the motorcycle was connected to the games controller accelerator pedal via the standard throttle cable. When the throttle on the motorcycle is applied the throttle cable pulls the spring loaded foot pedal down. The degree of movement can be calibrated to different ranges to represent different motorcycle throttle characteristics.

- braking - a limitation of the games controller is that the standard car brake pedal interface only offers a single braking input. On motorcycles using both the front and rear brake together or independently is a key riding skill and so an innovative solution was developed. Integrated and distributed ratios were developed so that the functional fidelity of the brakes is therefore preserved as the user is required to use both brakes to get the maximum braking effort (as would be expected on a real motorcycle).

- gear selection - the motorcycle gears are interfaced with the 'STISIM-Drive' software using two 'push to make' switches wired to auxiliary buttons on the gear selector unit. The device was designed and manufactured to operate as a real motorbike, and not interfere with the rider's natural riding position and gear changing action, and to have a minimal impact on the aesthetics of the motorcycle. 
- steering angle - the steering wheel was removed from games controller so that the steering column could be connected to the steering unit of the motorcycle. A bespoke design allows for an initial free rotation of the column to allow for the 'STISIM-Drive' $\pm 720^{\circ}$ steering calibration.

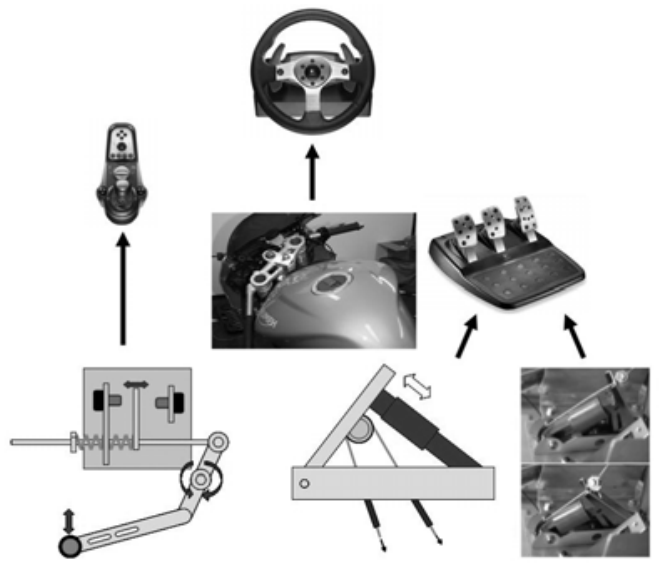

Fig. 2. Integrated motorcycle controls with an automotive games controller

\section{Using and Evaluating MotorcycleSim}

MotorcycleSim provided the basis for an in depth investigation of rider behaviour given its unique ability to test rider behaviour in a controlled environment [6]. Within a main riding scenario various sub-scenarios were developed and additional tasks were designed within the integrated experiment approach in order to explore a wide range of skills, attitudes and behaviours across three rider experience groups. The study was designed to compare Novice, Experienced and Advanced trained riders and the process adopted allowed for comprehensive data collection and analysis with minimal disruption to participants or biases creeping into the data [6]. By combining 'STISIM-Drive' and 'MotorcycleSim' this approach demonstrated the power and innovation of exploring a variety of rider attributes in a single investigation. Furthermore, and in line with the iterative principles of ISO:13407, an evaluation of MotorcycleSim has been conducted which have provided evidence to support the effectiveness of static simulation as well as ideas for future development [3].

\section{Conclusions}

MotorcycleSim is at the forefront of motorcycle simulation. No other simulator exists like it, that provides a full size, interactive, realistically controlled, accurate performance measurement platform which can be used to investigate motorcycle design, rider equipment, rider behaviour, road safety and rider training and competence. A major strength in its development has been the underlying user-centred approach in capturing 
user requirements at the earliest opportunity which have provided a basis for the initial design and onward development of MotorcycleSim. The potential of transport ergonomics in all its guises is vast and novel methods and procedures are likely to emerge in order to better understand the fine detail of road user behaviour. Simulated trials will continue to provide a valuable research tool for investigators and given the investment in technologies and equipment, it is often necessary to design integrated approaches that offer the greatest potential to capture data on many levels.

Acknowledgement. The authors would like to thank The Institute of Advanced Motorists, Phoenix Distribution (Arai helmets) and 'STISIM-Drive' for their support.

\section{References}

1. Stedmon, A.W., Brickell, E., Hancox, M., Noble, J., Rice, D.: MotorcycleSim: a usercentred approach for an innovative motorcycle simulator. In: Golightly, D., Rose, T., Bonner, J., Boyd Davis, S. (eds.) Creative Inventions \& Innovations for Everyday HCI. The Ergonomics Society, Leicester (2008)

2. Stedmon, A.W., Young, M., Hasseldine, B.: Keeping it real or faking it: The trials and tribulations or real road studies and simulator research in transport research. In: Bust, P. (ed.) Contemporary Ergonomics 2009. Taylor \& Francis, London (2009)

3. Stedmon, A.W., Hasseldine, B., Rice, D., Young, M., Markham, S., Hancox, M., Brickell, E., Noble, J.: MotorcycleSim: an evaluation of rider interaction with an innovative motorcycle simulator. The Computer Journal (2009), doi:10.1093/comjnl/bxp071

4. Earthy, J., Sherwood Jones, B., Bevan, N.: The improvement of human-centred processes: Facing the challenge and reaping the benefit of ISO:13407. International Journal of Human Computer Studies 55(4), 553-585 (2001)

5. Stone, R.J.: Virtual Reality. Virtual and Synthetic Environments: Technologies and Applications. In: Karkowski, W. (ed.) International Encyclopaedia of Ergonomics and Human Factors. Taylor \& Francis, London (2001)

6. Stedmon, A.W., Crundall, D., Crundall, E., Saikayasit, R., Ward, P., van Loon, E., Irune, A.A.: Advanced Training \& Rider Performance. University of Nottingham (2010) 